\title{
Molecular Dynamics Simulations on trans- and cis-Decalins: The Effect of Partial Atomic Charges and Adjustment of "Real Densities"
}

\author{
Roman Eremin \\ Laboratory of Radiation Biology, Joint Institute for Nuclear Research, Dubna 141980, Russia \\ $\&$ \\ University Center, Joint Institute for Nuclear Research, Dubna 141980, Moscow Region, Russia \\ E-mail: eremin_roman@inbox.ru \\ Kholmirzo Kholmurodov \\ Laboratory of Radiation Biology, Joint Institute for Nuclear Research, Dubna 141980, Russia
}

$\&$

International University “Dubna”, 141980, Dubna, Moscow Region, Russia

E-mail: mirzo@jinr.ru

Mikhail Avdeev

Frank Laboratory of Neutron Physics, Joint Institute for Nuclear Research, Dubna 141980, Russia

E-mail: avd@nf.jinr.ru

Viktor Petrenko

Frank Laboratory of Neutron Physics, Joint Institute for Nuclear Research, Dubna 141980, Russia

$\&$

Physics Department, Kyiv Taras Shevchenko National University, Kyiv 03022, Ukraine

E-mail: vip@nf.jinr.ru

Kenji Yasuoka

Department of Mechanical Engineering, Keio University, Yokohama 223-8522, Japan

E-mail: yasuoka@mech.keio.ac.jp

Received: December 2, $2011 \quad$ Accepted: December 13, $2011 \quad$ Published: February 1, 2012

doi:10.5539/ijc.v4n1p14

URL: http://dx.doi.org/10.5539/ijc.v4n1p14

\begin{abstract}
A molecular dynamics (MD) simulation of the organic compounds trans- and cis-decalin is performed with the adjustment of their experimentally observed densities. For a trans-decalin model system, the energy and structural properties are studied for different atomic charge distributions. The relationship between the main interaction forces (Coulombic and van der Waals) of the trans- and cis-decalin systems has been examined, and the status of the molecular forces governing the nature of the processes in the crystal or liquid phases has been established. The obtained results on the density peculiarities are interpreted in terms of a non-uniform charge distribution and van der Waals forces efficiently inhibiting the electrostatic ones. Possible applications of the obtained MD simulations results in magnetic fluid physics are discussed.
\end{abstract}


Keywords: Molecular dynamics, trans- and cis-Decalins, Van der Waals forces, Coulombic interactions

\section{Introduction}

Complex colloidal systems with controlled properties are currently studied in order to determine their specific structural features. Ferrofluids (FFs) are typical representatives of these systems. FFs are colloidal liquids made of nanoscale ferromagnetic or ferrimagnetic particles suspended in a carrier fluid (usually, an organic solvent or water). Their properties are close to those of the homogeneous liquids with a relatively high magnetic susceptibility (Shliomis, M. I., 1974; Rosensweig, R. E., 1985). A unique combination of such properties finds many applications in technology and engineering (Odenbach, S., 2003; Proc. of the 10th Intern. Conference on Magnetic Fluids, 2005). In recent years, possible biomedical applications of FFs have also been discussed with great interest (Fortin, J. P., 2007; Proc. of the 7th Intern. Conference on the Scientific and Clinical Applications of Magnetic Carriers, 2009). The magnetic particles are coated with a surfactant layer that prevents them from sticking together, stabilizing the whole system.

Benzene solutions of carboxylic acids (CAs), which can be used as a surfactant to produce FFs, were studied using the molecular dynamics method; and the results were compared with small-angle neutron scattering (SANS) and high-precision densitometry data. It is known that different CAs have different stabilizing abilities, which can be caused by differences in the solvent-acid interaction (Avdeev, M. V., Kholmurodov, Kh. T., Rus. J. Phys. Chem. A, 2009; Kholmurodov, Kh., Yasuoka, K., Natural Science, 2010). Decahydronaphthalene (decalin) can be used as a solvent for FFs; therefore, such interaction in this solvent is of current interest (Avdeev M. V. et al., 2011).

Decalin - a bicyclic organic compound - is an industrial solvent. It is a colorless liquid with an aromatic odor; it is used as a solvent for a number of organic compounds and polymers. Decalin exists in cis and trans isomeric forms differing only in the relative positions of the cyclic rings (Figure 1).

The trans form is energetically more stable. The boiling and melting points of this form are $185.5{ }^{\circ} \mathrm{C}$ and $-31.5{ }^{\circ} \mathrm{C}$, respectively. Its density is $0.87 \mathrm{~g} / \mathrm{cm}^{3}$ at $20{ }^{\circ} \mathrm{C}$. As for cis-decalin, the boiling and melting points are, respectively, $194.6{ }^{\circ} \mathrm{C}$ and $-43.2{ }^{\circ} \mathrm{C}$; the density is $0.897 \mathrm{~g} / \mathrm{cm}^{3}$ at $20{ }^{\circ} \mathrm{C}$ (Dean, J. A., 1999). In the industry, decalin can be prepared as a mixture of different forms through the naphthalene hydrogenation in the presence of a catalyst (Donaldson, N., 1958).

The density peculiarities of trans- and cis-decalins described above are supposed to be governed by well-known intermolecular forces. First of all, the relationship between the Coulomb and van der Waals interactions can determine the nature of the processes in the trans- and cis-decalin systems in their liquid states. Recent theoretical and experimental studies show that van der Waals interactions define a number of critical effects in ion-stabilizing FFs materials (Cerda, J., 2010). On the surface of FFs nanoparticles, the charge distribution can be non-uniform, which causes the residual van der Waals interactions to be strongly dominant. In many aspects, the van der Waals interactions can inhibit the Coulomb one. The competing Coulomb and van der Waals forces in diluted FFs considerably affect the system characteristics obtained by SANS. In this work, we aimed at determining the structural and energy properties of trans- and cis-decalins. First, using the MD method, we generated various atomic charge distributions (Coulombic interactions) in trans-decalin. The trans-decalin molecule is more symmetric, and its structural data are more informative than those of the cis-decalin one. Next, we estimated the Lennard-Jones parameters (van der Waals potential and forces) to provide the experimentally observed densities for both trans- and cis-decalins.

\section{Materials and Methods}

Molecular dynamics (MD) simulations on trans- and cis-decalin structures have been performed using DL_POLY 2.18 general-purpose code (Smith, W., 1996; Smith, W., 2008; Kholmurodov, K., Smith, W., Yasuoka, K., \& Ebisuzaki, T., 2000). Initially, a MD study was fulfilled on a trans-decalin system with the crystal structure that had been recently solved and refined with an X-ray powder diffraction technique (Eibl, S., 2009). In our present study, a trans-decalin model was constructed by describing each atomic position taking into account the periodic symmetry boundary conditions (Figure 2). The simulation cell consisted of a total of 700 decalin molecules. The system's spatial size was 55 × 53 × 53 angstroms $(7 \times 5 \times 10$ cells of the crystal structure). The decalin molecules were considered to be rigid units with constant bond lengths.

The temperature of the system was controlled through the Nose-Hoover (NVT) ensemble. The Verle integration algorithm for the numerical solution of the equations of motion was realized with the time step of $0.001 \mathrm{ps}$.

The intermolecular interactions were modeled with the Lennard-Jones (LJ) and Coulomb potentials (Allen, M. P., 1989): 


$$
U=\sum_{i j} 4 \varepsilon_{i j}\left[\left(\frac{\sigma_{i j}}{r_{i j}}\right)^{12}-\left(\frac{\sigma_{i j}}{r_{i j}}\right)^{6}\right]+\frac{q_{i} q_{j}}{r_{i j}},
$$

where the LJ (12-6) potential was cut off at $8 \AA$ of the spherical radii; the cutoff radius for the Coulomb interactions (for the charged systems) was $12 \AA$. For the non-identical atoms, the interaction parameters were found using the Lorentz-Berthelot mixing rule (Lorentz, H. A., 1881; Berthelot, D., 1889):

$$
\begin{aligned}
& \sigma_{i j}=\left(\sigma_{i i}+\sigma_{j j}\right) / 2, \\
& \varepsilon_{i j}=\sqrt{\varepsilon_{i i} \varepsilon_{j j}} .
\end{aligned}
$$

As for the statistical data, graphs of the radial distribution functions (RDF) of various atomic pairs $(\mathrm{C}-\mathrm{C}, \mathrm{C}-\mathrm{H}$, $\mathrm{H}-\mathrm{H}$ ) were built at the start and end of the simulations and compared. The RDF behaviors demonstrate the differences between the phase states; in general, the RDF reflects the peculiarities of the liquid and crystal states. The RDF $g(r)$ shows the time average influence of the presence of an atom on the positions of neighboring atoms; it is proportional to the probability of finding two atoms at a distance of $r+\Delta r$ from each other (Allen, M. P., 1989).

$$
\rho g(\vec{r})=\frac{1}{N}\left\langle\sum_{i}^{N} \sum_{j \neq i}^{N} \delta\left[\vec{r}-\vec{r}_{i j}\right]\right\rangle
$$

where $N$ is the total number of atoms, $\rho=N / V$ is the numerical density of atoms, $\mathrm{r}_{i j}$ is the vector between the centers of atoms $i$ and $j$, and angle brackets denote averaging over time. For distances shorter than one atomic diameter, $g(r)=0$. At larger distances in a liquid, an atom should not influence the positions of other atoms; that is, $g(r)=1$.

\section{Results}

\subsection{Intermolecular structure}

From the MD simulation data, we first built an RDF graph for the neutral (with zero partial atomic charges) trans-decalin system (Figure 3). The RDF $g(r)$ functions in Figure 3 correspond to the atomic pairs $\mathrm{C}-\mathrm{C}, \mathrm{H}-\mathrm{H}$, and $\mathrm{C}-\mathrm{H}$ at initial and final states of the system under simulation, respectively. In Figure 3, RDF graphs are shown for the initial and relaxed final states of the trans-decalin system. The RDF behavior can be related to trans-decalin's crystal and liquid phases. From Figure 3, it is seen that the $\mathrm{H}-\mathrm{H}$ pair's RDF reaches its maximum at a short distance of $1,5 \AA$, while the $\mathrm{C}-\mathrm{C}$ pair's $\mathrm{RDF}$, at 3,5 $\AA$. The $\mathrm{C}-\mathrm{C}$ pair's RDF has two maximums and a small minimum between them at $5 \AA$.

Next, we generated various artificial charge values in trans-decalin molecule (Table 2) corresponding to the C and $\mathrm{H}$ atoms - to describe the influence of the decalin charge distribution on the RDF behavior. The choice of the charge values in Table 2 was rather arbitrary, meeting only the molecule electroneutrality requirement. However, the distribution of the charge denoted ch-006 is close to that in real trans-decalin which was calculated by the ZINDO/1 semi-empirical method of quantum chemistry (Zerner, M., 1991).

The RDF results for different charge distributions described above were compared with those in Figure 3 for the neutral system. A comparison analysis shows that introducing a charge distribution like the ch-006, ch-01, ch-03, ch03, and ch04 does not almost change the RDF behavior for both crystal and liquid phases. As seen from Fig.4, starting from the ch05 and higher values, the artificial introduction of large partial charges produces visible changes in $g(r)$. This observation remains valid for all $\mathrm{C}-\mathrm{C}, \mathrm{C}-\mathrm{H}$, and $\mathrm{H}-\mathrm{H}$ atomic pairs. Thus, in the plausible charge ranges, the RDF does not undergo any visible changes, indicating that the electrostatic interactions would not necessarily be dominant over the van der Waals ones. Only increasing the partial atomic charge up to its limit value of 1 (ch08-ch10) leads to a significant change in $g(r)$ behavior (Figure 5).

\subsection{Configuration and energy profiles}

In Figure 6, configuration snapshots of a trans-decalin system are presented for the neutral and maximum partial charge distributions (ch00 and ch10, respectively) in their final relaxed states. For the neutral system (ch00), the average density is uniform; for large partial atomic charges (ch10), the liquid density is increasingly non-uniform as strong charge interactions cause local clusterization. Thus, the fluid of trans-decalin molecules with the zero 
charge (ch00) has a homogeneous structure; the comparison of its structural shape with the liquid in the case of a high non-zero charge (ch10) is straightforward (Figure 6: ch00 (left), ch10 (right)).

Next, we traced the evolution of the main energy characteristics of trans-decalin with an artificial change in the atomic charge distribution in the molecule. In Figure 7 and 8, MD simulation results are presented for the total internal and configuration energies of the system. First, it should be noted that all energy curves tend to constant levels indicating energy conservation irrespective to the charge values. An increase in the charge values leads to an increase in internal energy. Obviously, it can be explained as higher charge values generating stronger electrostatic interactions. Figure 8 shows a graph of the configuration energy of the system.

The configuration energy consists of two main components. The first one is the van der Waals interaction energy. Its behavior does not depend directly on the variation of the charge distributions up to ch05 (Figure 9). However, from the values of ch08 to ch10, the van der Waals energy curves strongly correlate with increasing the charge values. The second main component of the configuration energy is the electrostatic interaction energy (Figure 10), which underlies the fact that the partial atomic charge variations lead to an effective repulsion and attraction between the particles of the system. It is seen from Figure 10 that the electrostatic Coulomb interaction energy decreases and becomes negative with increasing a partial atomic charge. Comparing the behavior of the van der Waals and Coulomb potential energies is straightforward (Figure 9 and 10).

\subsection{Adjustment of "real densities"}

From the MD results presented above, we conclude that in the reasonable ranges of the partial atomic charge distributions (for example, the charge values obtained by accurate quantum chemistry calculations), the van der Waals interactions seem to be a dominant factor. The van der Waals forces can inhibit here the electrostatics ones. In other words, in the such systems (like trans- and cis-decalins), the correct description of the van der Waals potential is extremely important. In this section, MD simulation series have been performed aimed at the precise evaluation of the correct range of the LJ potential parameters for trans- and cis-decalins to ensure a good approximation of the van der Waals interaction in the system. The LJ parameters $\sigma_{\mathrm{H}}$ and $\sigma_{\mathrm{C}}$ have been measured with a NPT ensembles. The $\sigma_{\mathrm{H}}$ and $\sigma_{\mathrm{C}}$ have been multiply re-estimated in such a way that it is ensured that the densities of both trans- and cis-decalin are 0.87 and $0.897 \mathrm{~g} / \mathrm{cm}^{3}$, respectively - the values experimentally observed under normal conditions.

MD calculation results are summarized for trans- and cis-decalins in Figure 11 and 12, respectively, where configuration snapshots are shown along with density profiles defined through relaxations for NPT ensembles. The simulated and experimental values of the parameters $\sigma_{\mathrm{H}}$ and $\sigma_{\mathrm{C}}$ are shown in Figure 11 and 12. Crossing the MD-simulated density surfaces by the planes of the experimentally observed values (dotted lines), we find the unique and optimal LJ parameters, e.g. $\sigma_{\mathrm{H}}(\operatorname{sim})=.\sigma_{\mathrm{H}}($ exp. $)$ and $\sigma_{\mathrm{C}}(\operatorname{sim})=.\sigma_{\mathrm{C}}(\exp$.).

Thus, from Figure 11 and 12, we found the intersections of the simulated and experimental density planes, where we get the following LJ parameters: $\sigma_{\mathrm{H}}=2.693 \AA$ and $\sigma_{\mathrm{C}}=3.221 \AA$. It should be stressed that these values deviate within an error of less than $4 \%$ from the well-known ones, $\sigma_{\mathrm{H}}=2.81 \AA$ (Murad, S., 1978) and $\sigma_{\mathrm{C}}=3.35 \AA$ (Tildesley, D. J., 1981), which are widely used in MD simulation analysis (compare Ref. (Murad, S., 1978): $\sigma_{\mathrm{H}}=$ $2.81 \AA$ and $\varepsilon_{\mathrm{H}} / \mathrm{k}_{\mathrm{B}}=8.6 \mathrm{~K}$; Ref. (Tildesley, D. J., 1981): $\sigma_{\mathrm{C}}=3.35 \AA$ and $\varepsilon_{\mathrm{C}} / \mathrm{k}_{\mathrm{B}}=51.2 \mathrm{~K}$, where $\mathrm{k}_{\mathrm{B}}-$ Boltzmann's constant).

Finally, concerning LJ parameters estimation, it is well known that the parameter $\sigma_{\mathrm{C}}$ for carbon atom depends on its location within a molecule. As for the decalin molecule, its carbon atoms have to be $\mathrm{sp}^{3}$-hybridized, forming a tetragonal structure with the nearest atoms. The energy potential parameter $\varepsilon_{\mathrm{C}}$ for such carbon atom is 0.06 $\mathrm{kcal} / \mathrm{mol}$. Hence, the $\sigma_{\mathrm{C}}$ value was estimated from the results described before. Again, MD simulations have been performed to estimate the $\sigma_{\mathrm{H}}$ value of both trans- and cis-decalin to make sure that the density values are the same as observed experimentally. Calculation results for the $\sigma_{\mathrm{H}}$ parameter are shown in Figure 13 for transand cis-decalin (top and bottom, respectively). From Figure 13, we found that $\sigma_{\mathrm{H}}$ is $2.273 \AA$ for trans-decalin and $2.242 \AA$ for cis-decalin. The comparison of the values $\sigma_{\mathrm{H}}=2.273 \AA$ and $\varepsilon_{\mathrm{C}}=0.06 \mathrm{kcal} / \mathrm{mol}$ ( $\mathrm{sp}^{3}$-hybridization) with $\sigma_{\mathrm{H}}=2.693 \AA$ and $\varepsilon_{\mathrm{C}}=0.12 \mathrm{kcal} / \mathrm{mol}$ ( $\mathrm{sp}^{2}$-hybridization) is straightforward.

\section{Conclusion}

This study presents molecular dynamics (MD) models simulating trans- and cis-decalin solutions. The structural, energy and potential parameters have been defined in multiple series of MD calculations. First, the radial distribution function (RDF) graphs were built for the trans-decalin molecule and its behavior traced depending on changes in the artificial partial atomic charge distributions in the molecule. The charge distribution in the trans-decalin molecule has no significant effect on the $\mathrm{RDF}$ of the $\mathrm{C}-\mathrm{C}$ atomic pair within the reasonable charge 
range (including, the charges that can be predicted using quantum chemistry techniques). The trans-decalin fluid corresponding to the system with zero or low partial atomic charges has a homogeneous structure - contrary to the liquid model for a higher non-zero charge distribution. The crystal-liquid transitions are reflected in the RDF behavior for various charge distributions. The relationship between the main interaction forces of the system (Coulombic and van der Waals) has been examined, and the molecular forces governing the nature of the processes in the crystal or liquid phases have been cleared up. The Lennard-Jones (LJ) potential parameters have been precisely defined to ensure the correct description of the dominant van der Waals interactions. The use of the estimated LJ parameters yields the experimentally observed values of the liquid densities of both trans- and cis-decalin. It seems to be possible to describe the effect of the appearance of differences in the dispersion ability of different CAs in decalin by the MD simulation of these solutions from the structural point of view. For this purpose, the RDF of the atoms of the acid and decalin molecules for different acids should be carefully considered.

\section{Acknowledgments}

This work has been performed under joint collaboration projects between JINR (Russia), RIKEN (Japan), and Keio University (Japan). The work has been supported in part by Grant in Aid for the Global Center of Excellence Program for "Center for Education and Research of Symbiotic, Safe and Secure System Design" from Japan's Ministry of Education, Culture, Sport, and Technology. The MD simulations have been performed using computer software, hardware facilities, and cluster machines at the CICC (JINR), RICC (RIKEN), and Yasuoka Laboratory of Keio University (Japan). The authors would like to specially thank Mr. Sergei Negovelov (JINR) for technical assistance and helpful comments.

\section{References}

Allen, M. P., \& Tildesley, D. J. (1989). Computer Simulation of Liquids. Clarendon, Oxford, UK.

Avdeev, M. V., Aksenov, V. L., \& Feoktystov, A.V. (2011). On Determination of "Atomic" and "Magnetic" Sizes of Nanoparticles in Ferrofluids by Means of Small-Angle Neutron Scattering. JINR Preprint, P14-2011-22.

Avdeev, M. V., Kholmurodov, Kh. T., et al. (2009). The Determination of the Limiting Partial Molar Volume of Solutions of Monocarboxylic Acids in Benzene by Molecular Dynamics Simulation. Rus. J. Phys. Chem., 83, 1129-1133. http://dx.doi.org/10.1134/S0036024409070152

Berthelot, D. (1889). Sur le Melange des Gaz. Comptes Rendus de L'Academie des Sciences, 126, $1703-1706$.

Cerda, J. et al. (2010). Behavior of bulky ferrofluids in the diluted low-coupling regime: Theory and simulation. Phys. Rev. E, 81, 011501. http://dx.doi.org/10.1103/PhysRevE.81.011501

Dean, J. A. (1999). Lange's Handbook of Chemistry. McGraw-Hill, Inc., Chicago, IL, USA.

Donaldson, N. (1958). The chemistry and technology of naphthalene compounds. E. Arnold edition, London.

Eibl, S., Fitch A., Brunelli, M. et al. (2009). trans-Decahydronaphthalene (decalin) from powder diffraction data. Acta Cryst., 65, 278-280. http://dx.doi.org/10.1107/S0108270109013365

Fortin, J. P. et al. (2007). Size-Sorted Anionic Iron Oxide Nanomagnets as Colloidal Mediators for Magnetic Hyperthermia. J. Am. Chem. Soc., 129, 2628-2635. http://dx.doi.org/10.1021/ja067457e

Kholmurodov, Kh., Smith, W., Yasuoka, K., \& Ebisuzaki, T. (2000). Highly vectorised "link-cell” Fortran code for the DL_POLY molecular dynamics simulation package. Computer Physics Communications, 125(13), 167-192. http://dx.doi.org/10.1016/S0010-4655(99)00485-3

Kholmurodov, Kh., Yasuoka, K., et al. (2010). Molecular Dynamics Simulation of the Interaction of Carbon Nanotube and a Carbon Disulfide Solvent. Natural Science, 2(8), 902-910. http://dx.doi.org/10.4236/ns.2010.28111

Lorentz, H. A. (1881). Über die Anwendung des Satzes vom Virial in der kinetischen Theoric der Gase. Ann. Phys., 248, 127-136. http://dx.doi.org/10.1002/andp.18812480110

Murad, S., \& Gubbins, K. E. (1978). Molecular Dynamics Simulation of Methane Using a Singularity-Free Algorithm. Computer Modeling of Matter, ACS Symposium Series, 86, 62-71. http://dx.doi.org/10.1021/bk-1978-0086.ch005

Odenbach, S. (2003). Ferrofluids - Magnetically controllable Fluids and their Applications, Springer Lecture Notes in Physics, 594. 
Petrenko, V. I., Avdeev, M. V., Almasy, L. et al. (2009). Interaction of mono-carboxylic acids in benzene studied by small-angle neutron scattering. Colloid and Surfaces A., 337, 91-93. http://dx.doi.org/10.1016/j.colsurfa.2008.12.001

Proc. of the 10th Intern. Conference on Magnetic Fluids. (2005). J. Magn. Magn. Mater., 289, 1-544.

Proc. of the 7th Intern. Conference on the Scientific and Clinical Applications of Magnetic Carriers. (2009). J. Magn. Magn. Mater., 321, 1335-1688.

Rosensweig, R. E. (1985). Ferrohydrodynamics, Cambridge University Press, Cambridge, 357.

Shliomis, M. I. (1974). Magnetic fluids. Sov. Phys. Usp., 17, 153-169. http://dx.doi.org/10.1070/PU1974v017n02ABEH004332

Smith W., \& Forester, T. R. (1996). DL_POLY_2.0: A general-purpose parallel molecular dynamics simulation package. J. Molecular Graphics, 14, 136-141. http://dx.doi.org/10.1016/S0263-7855(96)00043-4

Smith, W., Forester, T. R., \& Todorov, I. T. (2008). The DL_poly 2 user manual. Version 2.18, STFC Daresbury Laboratory Daresbury, Warrington WA4 4AD Cheshire, UK.

Tildesley, D. J., \& Madden, P. A. (1981). An effective pair potential for liquid carbon disulphide. J. Mol. Phys., 42, 1137-1156. http://dx.doi.org/10.1080/00268978100100861

Zerner, M. (1991). Reviews in Computational Chemistry, Vol. 2, VCH, New York, 313. http://dx.doi.org/10.1002/9780470125793.ch8

Table1. The parameters of the Lennard-Jones potential

\begin{tabular}{|c|c|c|}
\hline & $\sigma, \AA$ & $\varepsilon, \mathrm{kcal} / \mathrm{mol}$ \\
\hline C-C & 3.30 & 0.12 \\
\hline H-H & 1.78 & 0.02 \\
\hline
\end{tabular}

Table 2. The different charge distributions for trans-decalin molecule

\begin{tabular}{|c|c|c|c|c|c|c|c|c|c|c|}
\hline Charge & ch00 & ch-006 & ch-01 & ch-03 & ch03 & ch04 & ch05 & ch08 & ch09 & ch10 \\
\hline $\mathrm{C}, \mathrm{e}^{-} \mid$ & +0.0 & -0.06 & -0.1 & -0.3 & +0.3 & +0.4 & +0.5 & +0.8 & +0.9 & +1.0 \\
\hline $\mathrm{H},\left|\mathrm{e}^{-}\right|$ & -0.0 & $+0.03(3)$ & $+0.05(5)$ & $+0.16(6)$ & $-0.16(6)$ & $-0.2(2)$ & $-0.27(7)$ & $-0.4(4)$ & -0.5 & $-0.5(5)$ \\
\hline
\end{tabular}

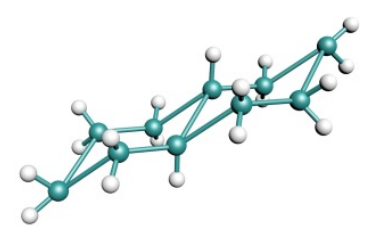

trans-decalin

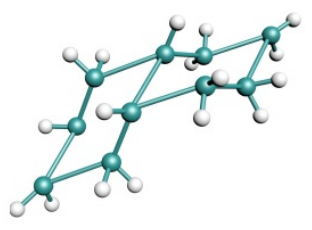

cis-decalin

Figure 1. The trans- and cis-decalin configurations

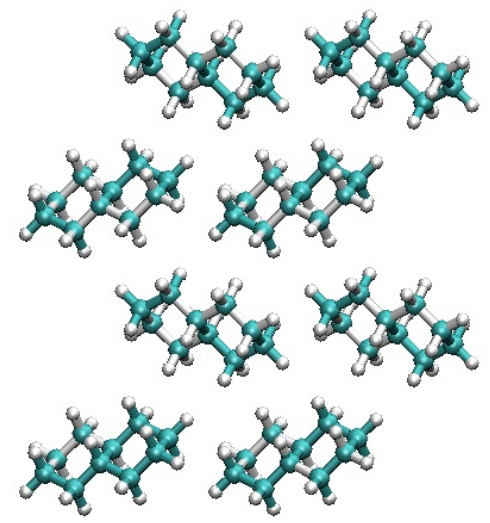

Figure 2. The crystal structure of trans-decalin 

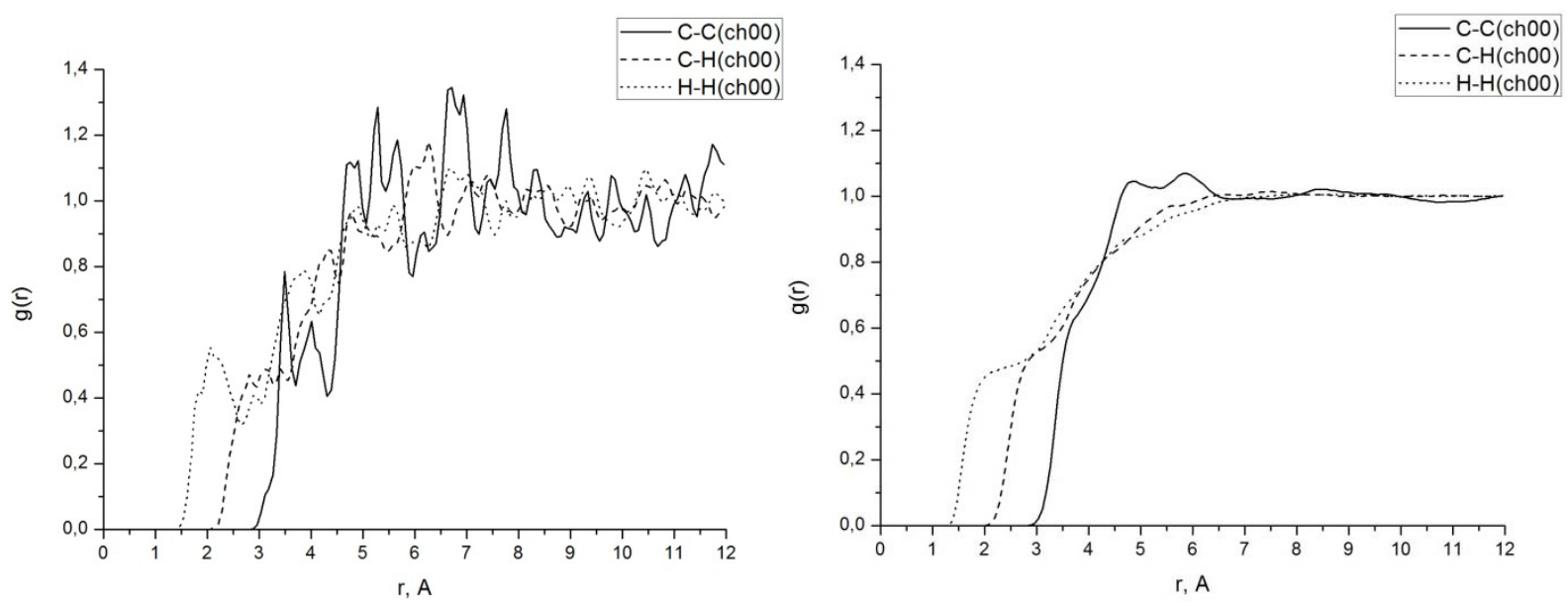

Figure 3. A comparison of $\mathrm{RDF}$ s for $\mathrm{C}-\mathrm{C}, \mathrm{C}-\mathrm{H}$, and $\mathrm{H}-\mathrm{H}$ atom pairs at the initial (left) and final (right) states
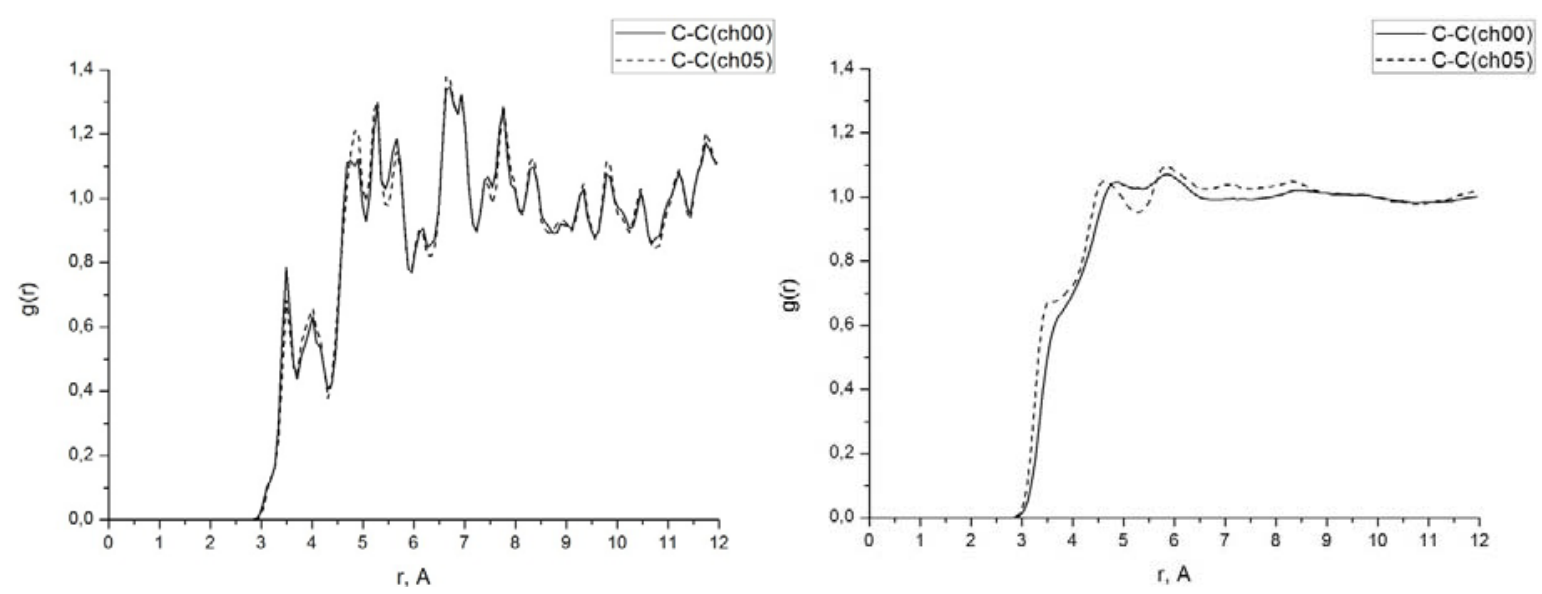

Figure 4. A comparison of $g[\mathrm{C}-\mathrm{C}]$ at the initial (left) and final (right) states for a system with ch00 and ch05 charge distributions
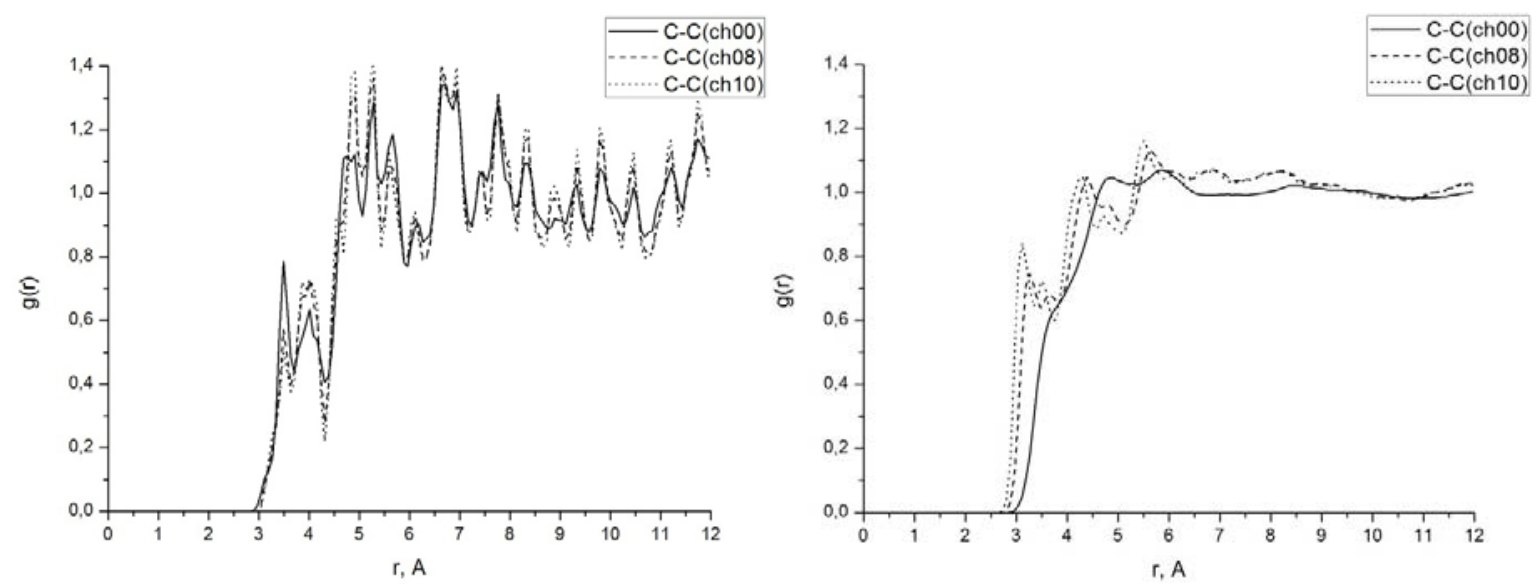

Figure 5. A comparison of $g[\mathrm{C}-\mathrm{C}]$ at the initial (left) and final (right) states for a system with ch00, ch08, and ch10 charge distributions 

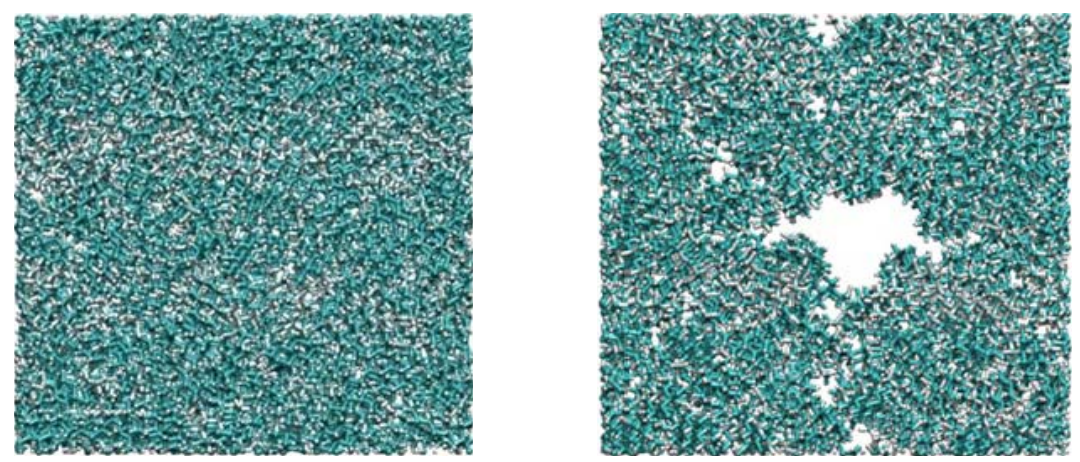

Figure 6. Snapshots of the system: ch00 (1) and ch10 (2) at relaxed final states

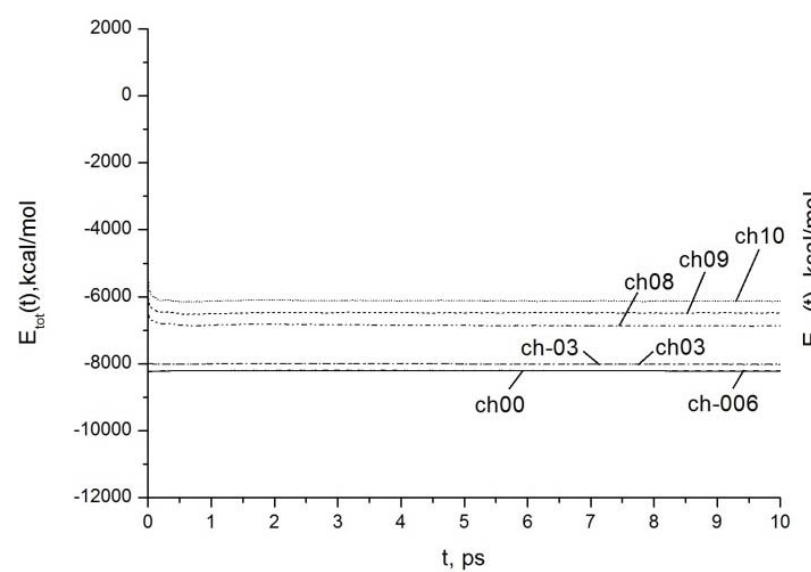

Figure 7. The total internal energy of the system

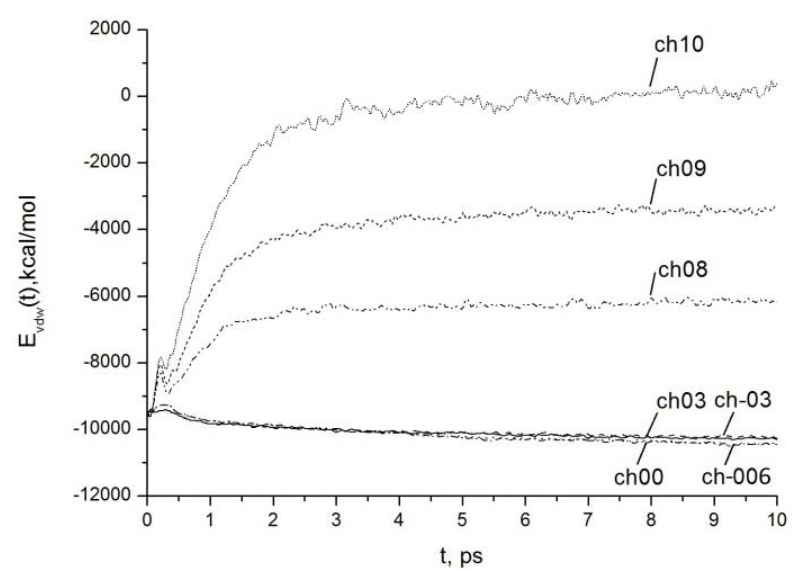

Figure 9. The van der Waals interaction energy

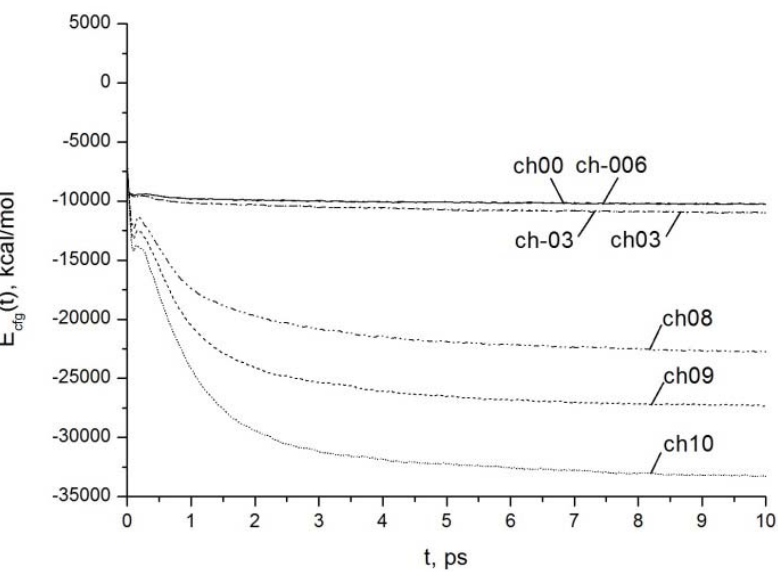

Figure 8 . The configuration energy of the system

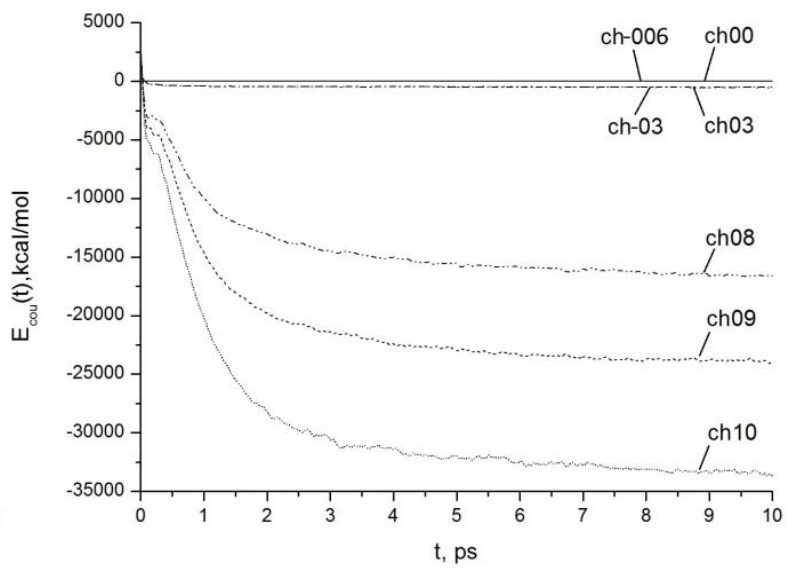

Figure 10. The electrostatic interaction energy 

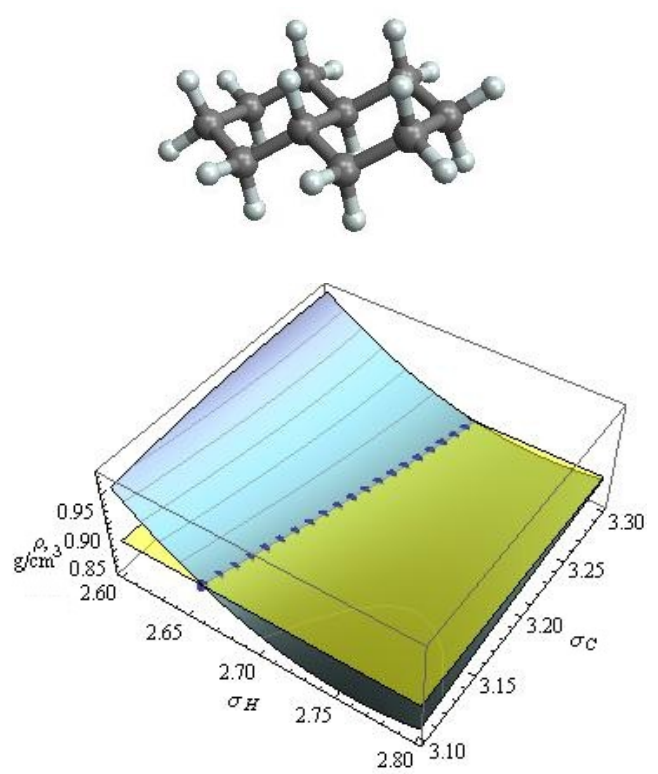

Figure 11. The trans-decalin molecule as balls and cylinders (top); the density profile after the system relaxation under normal conditions and a plane of $0.87 \mathrm{~g} / \mathrm{cm}^{3}$ (bottom)
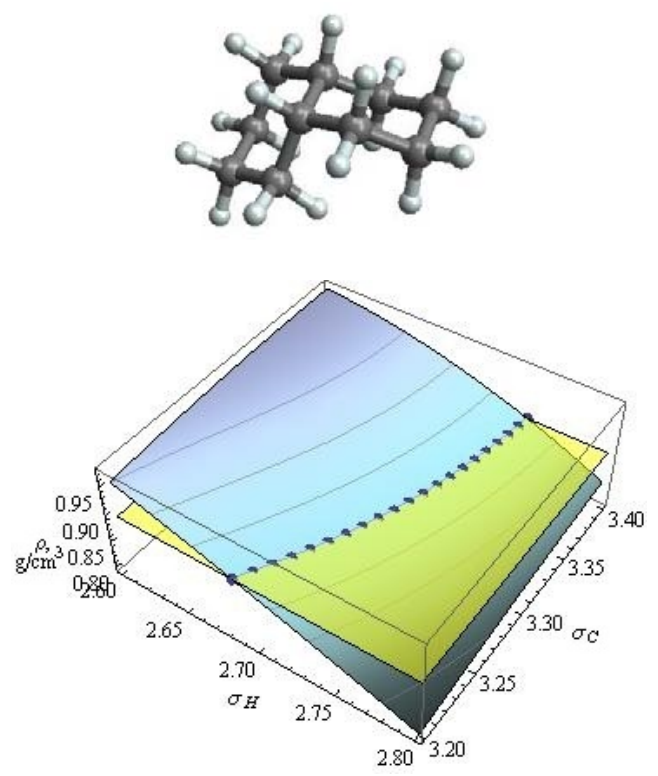

Figure 12. The cis-decalin molecule as balls and cylinders (top); the density profile after the system relaxation under normal conditions and a plane of $0.897 \mathrm{~g} / \mathrm{cm}^{3}$ (bottom)
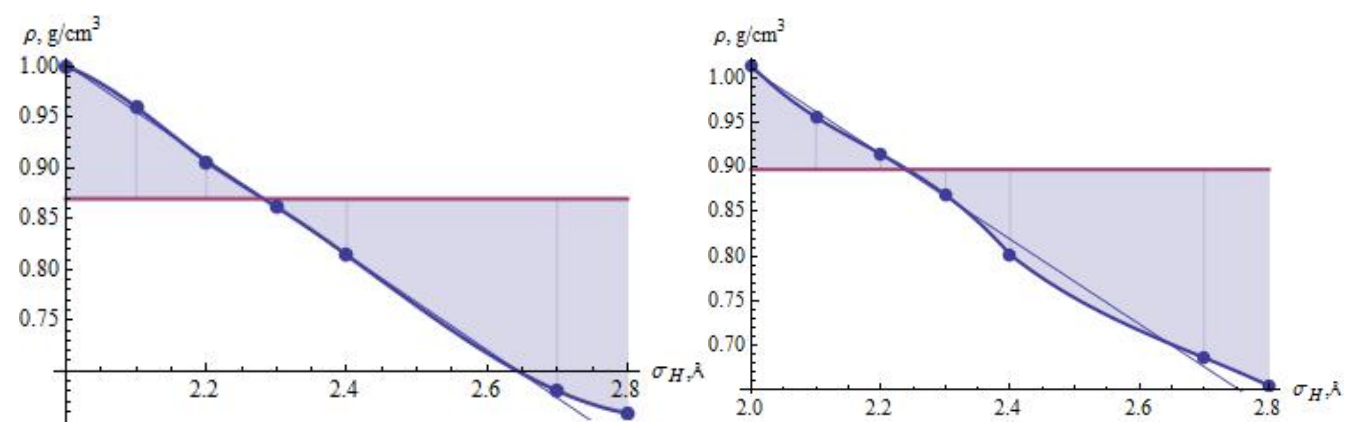

Figure 13. MD simulation results on $\sigma_{\mathrm{H}}$ for trans- (left) and cis-decalin (right) 